\title{
THE CLASS OF THE EXCEPTIONAL SETS FOR A GENERAL ASYMPTOTIC FORMULA
}

\author{
Danilo Bazzanella, Riccardo Camerlo
}

\begin{abstract}
We study the problem of the existence of a true exceptional set for an asymptotic formula, that is a minimal set - up to finite modifications - such that the asymptotic formula holds outside such a set. We give an analytic and a descriptive set theoretic characterisations for the existence of a true exceptional set, which we then apply by showing the non-existence of a true exceptional set in some well known situations. We prove in fact that, both from a category and a measure theoretic points of view, most asymptotic formulas do not have a true exceptional set.
\end{abstract}

Keywords: exceptional set, asymptotic formula, Borel set.

\section{Introduction}

In many mathematical problems it is useful to approximate the involved functions with other simpler functions that have the same asymptotic behavior. As usual we say that two functions, or sequences, $f$ and $g$ are asymptotic iff

$$
\lim _{x \rightarrow+\infty} \frac{f(x)}{g(x)}=1
$$

and we write $f \sim g$. We also write $f(x) \ll g(x)$ for $f=O(g)$.

It is often difficult to prove an asymptotic formula in this strong form and then we may restrict to the simpler problem of proving the validity of the asymptotic formula with an exceptional set. This means that there exists a set of positive numbers $S$, such that the relation (1.1) holds if $x \rightarrow+\infty$ through any sequence of numbers not belonging to $S$. In order to be of practical interest, it is often required that the measure of the set $S \cap[0, x]$ is negligible with respect to $x$ when $x \rightarrow+\infty$, namely

$$
\lim _{x \rightarrow+\infty} \frac{m(S \cap[0, x])}{x}=0 .
$$

2010 Mathematics Subject Classification: primary: 03E15, 11N37; secondary: 11N05 
In this case we say that the asymptotic formula holds for "almost all" values of $x$ (see A. Selberg [16]). The aim of this paper is to study the class of the exceptional sets for a general asymptotic formula. We will focus on the case of sequences (or arithmetic functions) $F: \mathbb{N} \rightarrow \mathbb{R}$.

We note that in many mathematical problems we have a different and simpler use of the exceptional sets. For example to deal with the Goldbach Conjecture we define the exceptional set as the set, supposedly empty, of the even numbers greater than 4 that can not be expressed as the sum of two primes. This exceptional set, as many others in number theory, is a single and well defined set. On the contrary, for every asymptotic formula in the form (1.1) we have an infinite class of the exceptional sets, as defined above, that could make the arguments more complicated, depending on whether this class has or not a minimum element (up to finite modifications), with respect to inclusion.

When this minimum exists we call it a true exceptional set of the asymptotic formula; otherwise the situation is more complicated and we need to deal with the entire class of the exceptional sets.

After some definitions and theoretic background presented in Sections 2 and 3, in Section 4 we provide two characterisations for the existence of a true exceptional set for an asymptotic formula. One characterisation is purely analytic and concerns the structure of limit points of the function; the other one is purely descriptive set theoretic and regards the complexity of the filter of exceptional sets. We establish that the equivalence relation $\simeq$ of having the same exceptional sets is Borel. We show moreover that the generic (in the sense of category) $F$ having 1 as a limit point does not admit a true exceptional set. Similarly almost all (in the sense of measure) $F$ having 1 as a limit point do not admit a true exceptional set. We end Section 4 by giving sufficient conditions for the non-existence of a true exceptional set.

When dealing with a concrete asymptotic formula, the ideal situation would be the existence of a true exceptional set. However, the results we obtain about the exceptional sets for some relevant asymptotic formulas of number theory provide further evidence that this is not quite a common situation, as the above category and measure theoretic arguments suggest. Indeed, in Section 5 we prove that the well known asymptotic formula about the distribution of primes in short intervals

$$
\pi(x+h(x))-\pi(x) \sim \frac{h(x)}{\log x}
$$

does not have a true exceptional set (and then that would be better in this case to talk about its class of the exceptional sets instead of its exceptional set). A similar result we prove in Section 6 about the asymptotic formula for the divisor function in short intervals

$$
\sum_{n<k \leqslant n+h(n)} d(k) \sim h(n) \log n,
$$

this one too without a true exceptional set. 


\section{Definitions and simple examples}

Let $F(n)$ an arithmetical function. To define the exceptional sets for the asymptotic formula $F \sim 1$, it is easier to start with their complements.

Definition 2.1. We shall denote by $\mathcal{I}_{F}$ the family of all $S \subseteq \mathbb{N}$ such that $\lim _{n \in S} F(n)=1$, meaning that $\forall \varepsilon \in \mathbb{R}^{+}, \exists N \in \mathbb{N}, \forall n \in S(n>N \Rightarrow|F(n)-1|<\varepsilon)$, so that for $S$ finite the property $\lim _{n \in S} F(n)=1$ is vacuously true.

A set $S \subseteq \mathbb{N}$ is exceptional for the asymptotic formula $F \sim 1$ (or, briefly, for $F$ ) if its complement is in $\mathcal{I}_{F}$; we shall denote the class of exceptional sets for $F$ by $\mathcal{S}_{F}$ :

$$
S \in \mathcal{S}_{F} \Longleftrightarrow \mathbb{N} \backslash S \in \mathcal{I}_{F} \Longleftrightarrow \lim _{n \notin S} F(n)=1 .
$$

This concept has been explicitly used for instance in [2].

Both $\mathcal{I}_{F}, \mathcal{S}_{F}$ are subsets of the Polish space $\mathcal{P}(\mathbb{N})$, and they can be studied in the framework of descriptive set theory — see A.S. Kechris [13] for a thorough introduction to the subject. This note is a contribution to the study of sets arising in number theory from a descriptive set theoretic perspective in the veine of articles like H. Ki and T. Linton [14]. To be as self-contained as possible, the next section gives a summary of the definitions and terminology needed in this paper.

Since the function $A \mapsto \mathbb{N} \backslash A$ is a homeomorphism of $\mathcal{P}(\mathbb{N})$, the complexities of $\mathcal{I}_{F}$ and of $\mathcal{S}_{F}$ are the same. Indeed, they are $\boldsymbol{\Pi}_{3}^{0}$ subsets of $\mathcal{P}(\mathbb{N})$.

Definition 2.2. On $\mathcal{P}(\mathbb{N})$, define the relation of almost equality $\equiv$ by letting, for $S, S^{\prime} \subseteq \mathbb{N}, S \equiv S^{\prime} \Longleftrightarrow\left(S \cup S^{\prime}\right) \backslash\left(S \cap S^{\prime}\right)=S \triangle S^{\prime}$ is finite.

In $\mathcal{P}(\mathbb{N}) / \equiv$, the order relation will be denoted $\subseteq^{*}$, while $\subset^{*}$ will stand for its strict part. Note that $\mathcal{I}_{F}$, thus $\mathcal{S}_{F}$ too, is invariant under $\equiv$, so the class of exceptional sets can be defined in the quotient $\mathcal{P}(\mathbb{N}) / \equiv$.

Definition 2.3. $\mathcal{S}_{F}^{*}=\mathcal{S}_{F} / \equiv$.

Note that

$$
\begin{aligned}
\mathcal{S}_{F}^{*}=\mathcal{S}_{G}^{*} & \Longleftrightarrow \mathcal{S}_{F}=\mathcal{S}_{G} \\
& \Longleftrightarrow \forall A \subseteq \mathbb{N}\left(\lim _{n \notin A} F(n)=1 \Leftrightarrow \lim _{n \notin A} G(n)=1\right) \\
& \Longleftrightarrow \forall A \subseteq \mathbb{N}\left(\lim _{n \in A} F(n)=1 \Leftrightarrow \lim _{n \in A} G(n)=1\right) .
\end{aligned}
$$

If $F, G$ satisfy this last relation, we write $F \simeq G$. Thus $\simeq$ is a coanalytic equivalence relation on $\mathbb{R}^{\mathbb{N}}$. We shall prove however in Theorem 4.8 that it is in fact a Borel equivalence relation. We also note that $\mathcal{I}_{F}$ is an ideal and consequently, $\mathcal{S}_{F}, \mathcal{S}_{F}^{*}$ are filters (in their respective domains). In fact, $\mathcal{I}_{F}$ is a P-ideal.

Definition 2.4. An ideal $\mathcal{I}$ consisting of countable subsets of a given set $X$ is a P-ideal if for any countable subfamily $\left\{I_{n}\right\}_{n \in \mathbb{N}}$ of $\mathcal{I}$ there exists $J \in \mathcal{I}$ such that $I_{n} \backslash J$ is finite for every $n$. The filter dual of a $\mathrm{P}$-ideal is a $\mathrm{P}$-filter. 
Definition 2.5. A true exceptional set for $F$ is, if it exists, a set $S \in \mathcal{S}_{F}$ such that for all $S^{\prime} \in \mathcal{S}_{F}, S \backslash S^{\prime}$ is finite.

In other words, the existence of a true exceptional set means that $\mathcal{S}_{F}^{*}$ is a principal filter. However, this is far from being a common situation, as it will be seen in Section 4. Let us illustrate this with some easy examples.

Example 2.6. The simplest case is when $\lim _{n \rightarrow+\infty} F(n)=1$. Then $\mathcal{I}_{F}=\mathcal{S}_{F}=\mathcal{P}(\mathbb{N})$ and $\emptyset$ is a true exceptional set.

Example 2.7. Let $A$ an infinite subset of $\mathbb{N}$. We define $F(n)=\left\{\begin{array}{ll}0 & \text { if } n \in A \\ 1 & \text { if } n \notin A\end{array}\right.$.

In this case $\mathcal{S}_{F}^{*}$ is the principal filter generated by $[A]_{\equiv}$, that is $A$ is a true exceptional set.

Example 2.8. For $k \geqslant 0$, let $A_{k}=\left\{n \in \mathbb{N}: n \equiv 2^{k} \bmod 2^{k+1}\right\}$ and observe that $A_{k} \cap A_{i}=\emptyset$ for every $k \neq i$ and

$$
\mathbb{N}=\bigcup_{k=0}^{+\infty} A_{k}
$$

Let $F(n)$ defined as $k /(k+1)$ for $n \in A_{k}$.

We prove that for every $[A]_{\equiv} \in \mathcal{S}_{F}^{*}$ there exists $[B]_{\equiv} \in \mathcal{S}_{F}^{*}$ such that $[B]_{\equiv} \subset^{*}$ $[A]_{\equiv}$ and this implies that the filter $\mathcal{S}_{F}^{*}$ is not principal. In order to prove the above assertion, we define the sequence $\left\{x_{k}\right\}$ choosing every $x_{k}$ such that $x_{k} \in A \cap A_{k}$. This is possible since $A \cap A_{k} \neq \emptyset$ for every $k$. By the definition of $x_{k}$ we have that

$$
\lim _{n \rightarrow+\infty} F\left(x_{n}\right)=1
$$

and then we obtain that $B=A \backslash\left\{x_{n}\right\}_{n \in \mathbb{N}} \in \mathcal{S}_{F}$ and $[B]_{\equiv \mathcal{C}^{*}}[A]_{\equiv}$.

\section{Descriptive set theoretic background}

This section contains basic descriptive set theoretic definitions, notations and facts that will be used in the sequel. A complete introduction to the subject is [13].

A subset $A$ of a topological space $X$ is:

- $\boldsymbol{\Pi}_{2}^{0}$, or $G_{\delta}$, if it is a countable intersection of open sets;

- $\boldsymbol{\Sigma}_{2}^{0}$, or $F_{\sigma}$, if it is a countable union of closed sets;

- $\Pi_{3}^{0}$, or $F_{\sigma \delta}$, if it is a countable intersection of $F_{\sigma}$ sets;

- Borel if it belongs to the $\sigma$-algebra of subsets of $X$ generated by the open sets. 
In evaluating the class which a given set belongs to, a useful tool is TarskiKuratowski algorithm, based on counting quantifiers in a given definition of the set.

A Polish space is a separable, completely metrisable topological space. A subspace of a Polish space is Polish, with the induced topology, if and only if it is a $G_{\delta}$ subset.

Given a class $\Gamma$ of subsets of Polish spaces, like the classes considered above, a subset $A$ of a Polish space $X$ is $\Gamma$-complete if it belongs to $\Gamma$ and for any subset $B$ of a zero-dimensional Polish space $Y$ which is in $\Gamma$ there is a continuous function $f: Y \rightarrow X$ such that $B=f^{-1}(A)$. Such a function $f$ is said to reduce $B$ to $A$. To prove that a given set $A$ in the class $\Gamma$ is $\Gamma$-complete it is enough to find an already known $\Gamma$-complete set $B$ and a continuous function $f$ reducing $B$ to $A$.

To mention the examples of complete sets we shall need, let us first recall a useful notation: the quantifiers $\exists^{\infty}, \forall^{\infty}$ over natural numbers will mean for infinitely many and for all but finitely many, respectively. Then:

- the family Fin of finite subsets of $\mathbb{N}$ is a $\Sigma_{2}^{0}$-complete subset of $\mathcal{P}(\mathbb{N})$; equivalently, the set $S_{2}=\left\{x \in\{0,1\}^{\mathbb{N}}: \forall^{\infty} n, x(n)=0\right\}$ of the eventually null binary sequences is a $\Sigma_{2}^{0}$-complete subset of $\{0,1\}^{\mathbb{N}}$;

- the set $P_{3}=\left\{x \in\{0,1\}^{\mathbb{N}^{2}}: \forall n, \forall^{\infty} k, x(n, k)=0\right\}$ of infinite $0-1$ matrices all of whose rows are eventually null is a $\Pi_{3}^{0}$-complete subset of $\{0,1\}^{\mathbb{N}^{2}}$.

A set is nowhere dense if its closure has empty interior. It is meagre if it is a countable union of nowhere dense sets. It is comeagre if its complement is meagre. Any dense $G_{\delta}$ subset of a Polish space is comeagre.

\section{Characterisations of the existence of a true exceptional set}

Definition 4.1. Let $\alpha \in \mathbb{R}$ and $F: \mathbb{N} \rightarrow \mathbb{R} . \alpha$ is a limit point of $F(n) \Longleftrightarrow$ there exists an increasing sequence of natural numbers $\left\{a_{n}\right\}$ such that $\lim _{n \rightarrow+\infty} F\left(a_{n}\right)=\alpha$.

Definition 4.2. We define $L_{F}$, the limit set of $F$, as the set of all limit points of the sequence $F(n)$. For $C \subseteq \mathbb{R}$, let also $\mathcal{F}_{C}=\left\{F \in \mathbb{R}^{\mathbb{N}}: C \subseteq L_{F}\right\}$.

If $1 \notin L_{F}$ then $\mathcal{S}_{F}^{*}=\left\{[\mathbb{N}]_{\equiv}\right\}$, and then we consider only the case $1 \in L_{F}$. Notice in fact the following.

Lemma 4.3. Given any $l \in \mathbb{R}$ the set $\mathcal{F}_{\{l\}}=\left\{F \in \mathbb{R}^{\mathbb{N}}: l \in L_{F}\right\}$ is a dense $G_{\delta}$ subset of $\mathbb{R}^{\mathbb{N}}$. In particular, the sequences $F$ with $1 \in L_{F}$ form a Polish space with the induced topology.

Proof. To see that the set is $G_{\delta}$, notice that it is the set of functions $F$ such that

$$
\forall \varepsilon \in \mathbb{Q}^{+}, \exists^{\infty} n \in \mathbb{N}, \quad|F(n)-l|<\varepsilon .
$$

Density holds as any finite sequence of real numbers can be extended to an element of $\mathcal{F}_{\{l\}}$. 
The following characterises the existence of a true exceptional set via the structure of the limit set.

Theorem 4.4. Let $F: \mathbb{N} \rightarrow \mathbb{R}$ and $1 \in L_{F}$. The two following conditions are equivalent:

(i) 1 is an isolated point of $L_{F}$;

(ii) F admits a true exceptional set.

Proof. In order to prove that (ii) implies (i) we assume that 1 is an accumulation point of $L_{F}$. Then we prove that for every $A \in \mathcal{S}_{F}$ there exists $B \in \mathcal{S}_{F}$ such that $[B]_{\equiv} \subset^{*}[A]_{\equiv}$ and then $\mathcal{S}_{F}^{*}$ is not a principal filter.

By the assumption that 1 is an accumulation point of $L_{F}$, we can build a sequence $\left\{\alpha_{k}\right\}$ such that $\alpha_{k} \in L_{F}, \alpha_{k} \neq 1$ and $\alpha_{k} \rightarrow 1$. Then there exists a sequence of natural numbers $\left\{a_{n}\right\}$, such that $F\left(a_{n}\right) \rightarrow \alpha_{1}$ and $a_{n} \notin A$ only for finitely many values of $n$. Then we can choose $y_{1} \in A$ such that $\left|\alpha_{1}-F\left(y_{1}\right)\right|<1$.

Further there exists a sequence of natural numbers $\left\{b_{n}\right\}$, such that $F\left(b_{n}\right) \rightarrow \alpha_{2}$ and $b_{n} \notin A$ only for finitely many values of $n$. Then we can choose $y_{2} \in A$ such that $y_{2}>y_{1}$ and $\left|\alpha_{2}-F\left(y_{2}\right)\right|<1 / 2$. Continuing in this way we define an increasing sequence $\left\{y_{k}\right\}$ such that $y_{k} \in A,\left|\alpha_{k}-F\left(y_{k}\right)\right|<1 / k$ and then $F\left(y_{k}\right) \rightarrow 1$.

Now we define $B=A \backslash\left\{y_{k}\right\}_{k \in \mathbb{N}}$. Observing that $B \in \mathcal{S}_{F}$ and $[B]_{\equiv} \subset^{*}[A]_{\equiv}$ we conclude that $\mathcal{S}_{F}^{*}$ is not a principal filter.

To prove the opposite implication, we suppose that there exists a real number $\varepsilon>0$ such that $(1-\varepsilon, 1+\varepsilon) \cap L_{F}=\{1\}$. Then we define

$$
A=\left\{n: F(n) \notin\left(1-\frac{\varepsilon}{2}, 1+\frac{\varepsilon}{2}\right)\right\}
$$

and observe that $A \in \mathcal{S}_{F}$. Now we note that every $B \in \mathcal{S}_{F}$ can only miss finitely many elements of $A$, and this concludes the proof of the theorem.

From this, we deduce a descriptive set theoretic characterisation of functions having a true exceptional set.

Corollary 4.5. Let $F: \mathbb{N} \rightarrow \mathbb{R}$.

(i) If $F$ admits a true exceptional set, then $\mathcal{S}_{F}$ is $\boldsymbol{\Sigma}_{2}^{0}$. Moreover, if $F$ does not converge to 1 , then $\mathcal{S}_{F}$ is $\boldsymbol{\Sigma}_{2}^{0}$-complete.

(ii) If $F$ does not admit a true exceptional set, then $\mathcal{S}_{F}$ is $\boldsymbol{\Pi}_{3}^{0}$-complete.

Proof. (i) Let $A$ be a true exceptional set of $F$. Then $B \in \mathcal{S}_{F} \Longleftrightarrow A \backslash B \in$ Fin. Since the operation $\backslash$ is continuous, the result follows. For the completeness part, since $A$ is infinite, let $A=\left\{a_{n}\right\}_{n \in \mathbb{N}}$ and consider the function $\mathcal{P}(\mathbb{N}) \rightarrow$ $\mathcal{P}(\mathbb{N}),\left\{x_{n}\right\}_{n \in \mathbb{N}} \mapsto\left\{a_{x_{n}}\right\}_{n \in \mathbb{N}}$. This reduces the $\boldsymbol{\Sigma}_{2}^{0}$-complete ideal of finite sets to $\mathcal{I}_{F}$.

(ii) In this case, $1 \in L_{F}$. Let $\varepsilon_{n}$ be a decreasing sequence of positive numbers such that for all $n$ there are infinitely many values $k$ with $\varepsilon_{n+1} \leqslant|F(k)-1|<$ $\varepsilon_{n}$. The existence of such a sequence is granted by Theorem 4.4. Enumerate $\left\{k \in \mathbb{N}: \varepsilon_{n+1} \leqslant|F(k)-1|<\varepsilon_{n}\right\}=\left\{a_{n h}\right\}_{h \in \mathbb{N}}$. Define the continuous function $f:\{0,1\}^{\mathbb{N}^{2}} \rightarrow \mathcal{P}(\mathbb{N})$ by $f(x)=\left\{a_{n h}: x(n, h)=1\right\}$. Then $f$ reduces the $\Pi_{3^{-}}^{0}$ complete set $P_{3}$ to $\mathcal{I}_{F}$. So both $\mathcal{I}_{F}, \mathcal{S}_{F}$ are $\boldsymbol{\Pi}_{3}^{0}$-complete. 
Corollary 4.6. If $F$ does not admit a true exceptional set, then $\mathcal{I}_{F}$ cannot be countably generated.

Proof. Each countably generated ideal is $\boldsymbol{\Sigma}_{2}^{0}$, directly from its definition.

The characterisation of corollary 4.5 suggests the following problem.

Problem 4.7. Is there a nice characterisation for those families of subsets of $\mathbb{N}$ that are filters of the exceptional sets for some real valued sequence $F$ ?

Notice that the (necessary) condition of being a $\boldsymbol{\Pi}_{3}^{0}$-complete P-filter containing all cofinite sets is not sufficient for being of the form $\mathcal{S}_{F}$, for some $F$ without a true exceptional set. Consider indeed the ideal $\mathcal{Z}$ of density 0 subsets of $\mathbb{N}$, that is the family of all sets $A \subseteq \mathbb{N}$ such that

$$
\lim _{n \rightarrow \infty} \frac{\operatorname{card}(A \cap\{1, \ldots, n\})}{n}=0 .
$$

By [14], this is $\boldsymbol{\Pi}_{3}^{0}$-complete. Moreover, it is a P-ideal. Indeed, let $\left\{J_{j}\right\}_{j \in \mathbb{N}} \subseteq \mathcal{Z}$. Let $m_{j}$ be such that

$$
\forall k>m_{j}, \quad \frac{\operatorname{card}\left(J_{j} \cap\{1, \ldots, k\}\right)}{k}<\frac{1}{2^{j}}
$$

and set $J=\bigcup_{j \in \mathbb{N}}\left\{k \in J_{j} \mid k>m_{j}\right\}$. In order to show $J \in \mathcal{Z}$, fix $n \in \mathbb{N}$. Let $\bar{m}$ be such that

$$
\forall j \in\{1, \ldots, n\}, \forall k>\bar{m}, \quad \frac{\operatorname{card}\left(J_{j} \cap\{1, \ldots k\}\right)}{k}<\frac{1}{2^{n}} .
$$

Then, for $k>\bar{m}$,

$$
\frac{\operatorname{card}(J \cap\{1, \ldots, k\})}{k} \leqslant \sum_{j \in \mathbb{N}} \frac{\operatorname{card}\left(J_{j} \cap\{1, \ldots, k\}\right)}{k} \leqslant \frac{n}{2^{n}}+\frac{1}{2^{n}}=\frac{n+1}{2^{n}} .
$$

As $n$ is arbitrary, it follows that $J \in \mathcal{Z}$. However, $\mathcal{Z}$ is not of the form $\mathcal{I}_{F}$ for any $F \in \mathbb{R}^{\mathbb{N}}$, since every infinite set of natural numbers has an infinite subset belonging to $\mathcal{Z}$.

Theorem 4.8. $\simeq$ is a Borel equivalence relation .

Proof. It is enough to consider the restriction of $\simeq$ to the $G_{\delta}$ set of all $F \in \mathbb{R}^{\mathbb{N}}$ with $1 \in L_{F}$, since the complement of such set is a single equivalence class. This set can in turn be split according to whether 1 is isolated in $L_{F}$ or not, as these two cases too are invariant under $\simeq$.

Case 1. The Borel condition for 1 to be isolated in $L_{F}$ is

$$
\exists \varepsilon>0, \forall \delta<\varepsilon, \forall^{\infty} n \quad(|F(n)-1|<\varepsilon \Rightarrow|F(n)-1| \leqslant \delta) .
$$

So for such an $F$ let $\varepsilon_{F}$ be the first positive rational, in some fixed enumeration, satisfying the condition; the function $F \mapsto \varepsilon_{F}$ is Borel. Then let $A_{F}=\{n \in \mathbb{N}$ : $\left.|F(n)-1|<\varepsilon_{F}\right\}$. Thus $F \simeq G$ if and only if $A_{F} \triangle A_{G}$ is finite, which is a Borel condition. 
Case 2. Fix a decreasing sequence $\varepsilon_{n}$ converging to 0 .

For each $F$ such that $1 \in L_{F}$ and 1 is a limit point of $L_{F}$, define an infinite matrix $M_{F} \in \mathbb{N}^{\mathbb{N}^{2}}$ as follows. First, if $n_{1}<n_{2}<\ldots$ are the (finitely or infinitely many) elements on which $F$ takes value 1 , enumerate such elements in the first column of $M_{F}$, that is let $M_{F}(l, 1)=n_{l}$.

Let $i_{1}^{F}$ be least such that $\left\{h \in \mathbb{N}:|F(h)-1| \geqslant \varepsilon_{i_{1}^{F}}\right\}$ is infinite and let $M_{F}(1, m)$ - for $m>1$ or $m$ ranging over $\mathbb{N}$ according to whether there exists at least one element on which $F$ equals 1 or not - be the increasing enumeration of this set. Given $i_{l}^{F}$, let $i_{l+1}^{F}$ be least such that $\left\{h \in \mathbb{N}: \varepsilon_{i_{l+1}^{F}} \leqslant|F(h)-1|<\varepsilon_{i_{l}^{F}}\right\}$ is infinite; let $M_{F}(l+1, m)$ be the increasing enumeration of this set, for $m>1$ or $m \in \mathbb{N}$ according to whether there are at least $l+1$ numbers on which $F$ equals 1 or not.

Notice that each natural number appears exactly once in $M_{F}$ and that $\lim _{n \in A} F(n)=1 \Longleftrightarrow \forall l, \forall^{\infty} m, M_{F}(l, m) \notin A$. This entails that, given $F, G$ according to this case, $F \simeq G$ if and only if

for all $l$ and for all infinite subsets $A$ of $\left\{M_{F}(l, m)\right\}_{m \in \mathbb{N}}$, there is $h$ such that $A \cap\left\{M_{G}(h, m)\right\}_{m \in \mathbb{N}}$ is infinite

and

for all $l$ and for all infinite subsets $A$ of $\left\{M_{G}(l, m)\right\}_{m \in \mathbb{N}}$, there is $h$ such that $A \cap\left\{M_{F}(h, m)\right\}_{m \in \mathbb{N}}$ is infinite.

Condition (4.1) is equivalent to ask that for all $l \in \mathbb{N}$ there is some $h \in \mathbb{N}$ such that

$$
\left\{M_{F}(l, m)\right\}_{m \in \mathbb{N}} \subseteq \bigcup_{i=1}^{h}\left\{M_{G}(i, m)\right\}_{m \in \mathbb{N}},
$$

which is a Borel condition, since the function $F \rightarrow M_{F}$ is Borel. Similarly for (4.2).

Next Theorems 4.10 and 4.13 establish that sequences admitting a true exceptional set are very uncommon, from both the points of view of category and of measure.

Lemma 4.9. For any $C \subseteq \mathbb{R}$, the set $\mathcal{F}_{C}$ is a comeagre $G_{\delta}$ subset of $\mathbb{R}^{\mathbb{N}}$.

Proof. If $C$ is non-empty, fix an at most countable dense subset $\left\{c_{n}\right\}_{n \in \mathbb{N}} \subseteq C$. Then observe that $\mathcal{F}_{C}=\bigcap_{n \in \mathbb{N}} \mathcal{F}_{\left\{c_{n}\right\}}$. Apply Lemma 4.3.

Theorem 4.10. The set of those functions $F$ such that $1 \in L_{F}$, but $F$ does not admit a true exceptional set is comeagre in $\mathbb{R}^{\mathbb{N}}$.

Proof. Fix any $C \subseteq \mathbb{R}$ having 1 as an accumulation point. Then apply Lemma 4.9 and Theorem 4.4.

Lemma 4.11. Let $\rho$ be any probability measure on $\mathbb{R}$ assigning positive measure to all open sets and let $\mu$ be the product measure on $\mathbb{R}^{\mathbb{N}}$. Then $\mu\left(\mathcal{F}_{\{l\}}\right)=1$ for any $l \in \mathbb{R}$. 
Proof. Indeed, $F \notin \mathcal{F}_{\{l\}} \Longleftrightarrow \exists \varepsilon, \forall^{\infty} n,|F(n)-l| \geqslant \varepsilon$. For any fixed $\varepsilon>0$, the set of $F \in \mathbb{R}^{\mathbb{N}}$ such that $\forall^{\infty} n,|F(n)-l| \geqslant \varepsilon$ is $\mu$-null. So $\mathbb{R}^{\mathbb{N}} \backslash \mathcal{F}_{\{l\}}$ is a countable union of null sets, and it is null too.

Corollary 4.12. If $\mu$ is as in Lemma 4.11, then $\mu\left(\mathcal{F}_{C}\right)=1$ for any $C \subseteq \mathbb{R}$.

Proof. As for Lemma 4.9, but using Lemma 4.11.

Theorem 4.13. The set of those functions $F$ such that $1 \in L_{F}$, but $F$ does not admit a true exceptional set has measure 1 in $\mathbb{R}^{\mathbb{N}}$ with respect to any product of a probability measure on $\mathbb{R}$ that is positive on each open set.

Proof. As for Theorem 4.10, but using corollary 4.12 instead of Lemma 4.9.

Theorem 4.4 allows to establish also the following two criteria for the nonexistence of a true exceptional set that will be applied later on.

Theorem 4.14. Let $F: \mathbb{N} \rightarrow \mathbb{R}, 1 \in L_{F}$,

$$
\lim _{n \rightarrow+\infty} F(n) \neq 1
$$

and

$$
\lim _{n \rightarrow+\infty}[F(n+1)-F(n)]=0 .
$$

Then $F$ does not have a true exceptional set.

Proof. From (4.3) it follows that

$$
\liminf _{n \rightarrow+\infty} F(n)<1
$$

or

$$
\limsup _{n \rightarrow+\infty} F(n)>1 .
$$

We suppose $m=\liminf _{n \rightarrow+\infty} F(n)<1$. The proof is similar if $\limsup _{n \rightarrow+\infty} F(n)>1$. From (4.5) and $1 \in L_{F}$ it follows that there exist two sequences of natural numbers $\left\{a_{n}\right\}$ and $\left\{b_{n}\right\}$ such that

$$
\lim _{n \rightarrow+\infty} F\left(a_{n}\right)=m<1
$$

and

$$
\lim _{n \rightarrow+\infty} F\left(b_{n}\right)=1 .
$$

Without loss of generality we may suppose $a_{n}<b_{n}<a_{n+1}$ for every $n$. Let $\lambda$ a real number such that $m<\lambda<1$. Then (4.4) implies that, for sufficiently large $n$, we have

$$
F\left(a_{n}\right)<\lambda-M_{n}<\lambda<\lambda+M_{n}<F\left(b_{n}\right)
$$

where

$$
M_{n}=\max _{a_{n} \leqslant k<b_{n}}|F(k+1)-F(k)| .
$$


Hence there exists a sequence of natural numbers $\left\{c_{n}\right\}$ such that $a_{n} \leqslant c_{n} \leqslant b_{n}$ and

$$
\lambda-M_{n}<F\left(c_{n}\right)<\lambda+M_{n},
$$

for sufficiently large $n$. This implies that

$$
\lim _{n \rightarrow+\infty} F\left(c_{n}\right)=\lambda
$$

and then $L_{F} \supset[m, 1]$. By Theorem 4.4 it follows that $F$ does not have a true exceptional set.

In the same way we can also prove the following.

Theorem 4.15. Let $F: \mathbb{N} \rightarrow \mathbb{R}$,

$$
\liminf _{n \rightarrow+\infty} F(n)<1<\limsup _{n \rightarrow+\infty} F(n)
$$

and

$$
\lim _{n \rightarrow+\infty}[F(n+1)-F(n)]=0 .
$$

Then $1 \in L_{F}$ and $F$ does not have true exceptional sets.

\section{The distribution of prime numbers in short intervals}

Let $\pi(x)$ be the prime-counting function that gives the number of primes less than or equal to $x$, for any real number $x$. The asymptotic law of the distribution of prime numbers was obtained independently in 1896 by Hadamard and de la Vallée-Poussin, who proved

$$
\lim _{x \rightarrow+\infty} \frac{\pi(x)}{x / \log x}=1
$$

This estimate is known as the Prime Number Theorem and implies that

$$
\pi(x+h(x))-\pi(x) \sim \frac{h(x)}{\log x},
$$

for any $x \ll h(x) \ll x$. Intervals of type $[x, x+h(x)]$, with $h(x) / x \rightarrow 0$ for $x \rightarrow+\infty$, are called "short intervals" and the distribution of prime numbers in such intervals is a very important problem in number theory.

Assume throughout this section that $h(x) / x \rightarrow 0$ for $x \rightarrow+\infty$. The best known unconditional result about the validity of the asymptotic formula (5.1) is due to M. N. Huxley [7] and asserts that it holds for $h(x)=x^{\theta}$ with $\theta>7 / 12$, which was slightly improved by D. R. Heath-Brown [6] to $\theta \geqslant 7 / 12-\varepsilon(x)$, for every $\varepsilon(x) \rightarrow 0$. Besides, Huxley's zero density estimate [7], in conjunction with the method of A. Selberg [16], show that (5.1) holds for almost all $x$ with $\theta>1 / 6$, which was slightly improved by A. Zaccagnini [17] to $\theta \geqslant 1 / 6-\varepsilon(x)$, for every $\varepsilon(x) \rightarrow 0$. 
If we assume some well-known heuristic hypotheses we can prove the validity of the asymptotic formula for smaller $\theta$. For instance A. E. Ingham [9, Theorem 4] proved that the asymptotic formula (5.1) holds for $\theta>1 / 2$, assuming the Lindelöf hypothesis, which states that the Riemann Zeta-function satisfies

$$
\zeta(\sigma+i t) \ll t^{\eta} \quad\left(\sigma \geqslant \frac{1}{2}, t \geqslant 2\right)
$$

for any $\eta>0$, which was improved by D. Bazzanella [3] who proved the same result assuming in turn two different hypotheses, both weaker than the Lindelöf hypothesis.

At last, under the assumption of the Riemann Hypothesis, it is known that (5.1) holds with $h(x) / x^{1 / 2} \log x \rightarrow+\infty$, and holds for almost all $x$ with $h(x) / \log ^{2} x \rightarrow$ $+\infty$, see A. Selberg [16].

This results reinforced the conjecture that the prime numbers are regularly distributed in very short intervals, even for $h(x)$ of the order of the power of logarithm. In $1985 \mathrm{H}$. Maier [15] proved a very notable result and disproved the strong assumptions about the regularity of the distribution of prime numbers, proving that

$$
\liminf _{x \rightarrow+\infty} \frac{\pi(x+h(x))-\pi(x)}{h(x) / \log x}<1<\limsup _{x \rightarrow+\infty} \frac{\pi(x+h(x))-\pi(x)}{h(x) / \log x},
$$

with $h(x)=\log ^{a} x$ and $a>1$.

This leads to consider the functions

$$
F(n)=\frac{\pi\left(n+\log ^{a} n\right)-\pi(n)}{(\log n)^{a-1}},
$$

with $a>1$, and the related asymptotic formula

$$
F(n) \sim 1 .
$$

For this functions we have

$$
\begin{aligned}
(F(n+1)-F(n)) \log ^{a-1}(n+1)= & \pi\left(n+1+\log ^{a}(n+1)\right)-\pi(n+1) \\
& -\left(1+o\left(\frac{1}{n}\right)\right)\left(\pi\left(n+\log ^{a} n\right)-\pi(n)\right) \ll 1,
\end{aligned}
$$

since $\pi\left(n+1+\log ^{a}(n+1)\right)-\pi\left(n+\log ^{a} n\right)$ is bounded. From this follows

$$
F(n+1)-F(n) \ll \frac{1}{\log ^{a-1}(n+1)}=o(1)
$$

and then (4.8) holds. From Theorem 4.15, we obtain that $\mathcal{S}_{F}^{*}$ is not a principal filter. In other words, there is not a true exceptional set. 
We can follow the same arguments if we suppose that the asymptotic formula (5.1) does not hold with $h(x)=x^{\theta}$, for some positive $\theta<7 / 12$. Let

$$
F(n)=\frac{\pi\left(n+n^{\theta}\right)-\pi(n)}{n^{\theta} / \log n} .
$$

We can prove (4.8) here as done for the preceding function. Indeed,

$$
\begin{aligned}
(F(n+1)-F(n)) \frac{(n+1)^{\theta}}{\log (n+1)}= & \pi\left(n+1+(n+1)^{\theta}\right)-\pi(n+1) \\
& -\left(1+O\left(\frac{1}{n}\right)\right)\left(\pi\left(n+n^{\theta}\right)-\pi(n)\right) \ll 1,
\end{aligned}
$$

since $n+1+(n+1)^{\theta}-\left(n+n^{\theta}\right)$ is bounded, which implies

$$
F(n+1)-F(n) \ll \frac{\log (n+1)}{(n+1)^{\theta}}=o(1) .
$$

If $1 \in L_{F}$, then Theorem 4.14 is applicable. Otherwise, to use Theorem 4.15 it remains only to verify (4.7). We proceed by reductio ad absurdum. We assume that (4.7) does not hold, that is

$$
\liminf _{n \rightarrow+\infty} F(n)>1 \quad \text { or } \quad \limsup _{n \rightarrow+\infty} F(n)<1 .
$$

This implies that

$$
\pi\left(n+n^{\theta}\right)-\pi(n)-\frac{n^{\theta}}{\log n} \gg \frac{n^{\theta}}{\log n}
$$

and then

$$
J(X, \theta)=\sum_{X \leqslant n \leqslant 2 X}\left|\pi\left(n+n^{\theta}\right)-\pi(n)-\frac{n^{\theta}}{\log n}\right|^{2} \gg \frac{X^{2 \theta+1}}{\log ^{2} X} .
$$

On the other hand we have

$$
\begin{aligned}
J(X, \theta) & =\sum_{X \leqslant n \leqslant 2 X} \int_{n}^{n+1}\left|\pi\left(n+n^{\theta}\right)-\pi(n)-\frac{n^{\theta}}{\log n}\right|^{2} \mathrm{~d} x \\
& =\sum_{X \leqslant n \leqslant 2 X} \int_{n}^{n+1}\left|\pi\left(x+x^{\theta}\right)-\pi(x)-\frac{x^{\theta}}{\log x}+O(1)\right|^{2} \mathrm{~d} x \\
& \ll \frac{1}{\log ^{2} X} \sum_{X \leqslant n \leqslant 2 X} \int_{n}^{n+1}\left|\left(\pi\left(x+x^{\theta}\right)-\pi(x)\right) \log x-x^{\theta}+O(\log x)\right|^{2} \mathrm{~d} x .
\end{aligned}
$$

Let $\vartheta(x)$ denote the Chebyshev function defined by the equation

$$
\vartheta(x)=\sum_{p \leqslant x} \log p,
$$


where $p$ runs over all primes $\leqslant x$ and observe that

$$
\begin{aligned}
\left|\left(\pi\left(x+x^{\theta}\right)-\pi(x)\right) \log x-\left(\vartheta\left(x+x^{\theta}\right)-\vartheta(x)\right)\right| \\
=\left|\sum_{x<p \leqslant x+x^{\theta}} \log x-\sum_{x<p \leqslant x+x^{\theta}} \log p\right| \\
=\sum_{x<p \leqslant x+x^{\theta}} \log \left(\frac{p}{x}\right) \ll \sum_{x<p \leqslant x+x^{\theta}} x^{\theta-1} \ll x^{2 \theta-1} .
\end{aligned}
$$

This in conjunction with (5.3), imply that

$J(X, \theta)$

$$
\begin{aligned}
& \ll \frac{1}{\log ^{2} X} \sum_{X \leqslant n \leqslant 2 X} \int_{n}^{n+1}\left|\vartheta\left(x+x^{\theta}\right)-\vartheta(x)-x^{\theta}+O(\log x)+O\left(x^{2 \theta-1}\right)\right|^{2} \mathrm{~d} x \\
& \ll \frac{1}{\log ^{2} X} \int_{X}^{2 X}\left|\vartheta\left(x+x^{\theta}\right)-\vartheta(x)-x^{\theta}\right|^{2} \mathrm{~d} x+X+\frac{X^{4 \theta-1}}{\log ^{2} X}+X^{2 \theta} .
\end{aligned}
$$

Under the assumption of the Riemann Hypothesis, this implies

$$
J(X, \theta) \ll X^{1+\theta}
$$

for every positive $\theta<7 / 12$, since it is well known that

$$
\int_{X}^{2 X}\left|\vartheta\left(x+x^{\theta}\right)-\vartheta(x)-x^{\theta}\right|^{2} \mathrm{~d} x \ll X^{1+\theta} \log ^{2} X
$$

for every positive $\theta$, see A. Selberg [16]. The bound (5.4) is in contradiction with (5.2) for every positive $\theta$ and then (4.7) holds. Unless to conflict with the Riemann Hypothesis, this implies that if the asymptotic formula

$$
\pi\left(n+n^{\theta}\right)-\pi(n) \sim \frac{n^{\theta}}{\log n}
$$

does not hold then does not have a true exceptional set.

\section{The divisor function in short intervals}

As usual, let

$$
\Delta(x)=\sum_{k \leqslant x} d(k)-x(\log x+2 \gamma-1)
$$

denote the error term in the Dirichlet divisor problem, where $d(k)$ is the number of divisors of $k$ and $\gamma$ is the Euler-Mascheroni constant. The current best upper bound for the error term $\Delta(x)$ is due to M. N. Huxley [8] who showed that for every $\varepsilon>0$ we have

$$
\Delta(x) \ll x^{131 / 416+\varepsilon} .
$$


The above estimate implies

$$
\sum_{n<k \leqslant n+h(n)} d(k) \sim h(n) \log n,
$$

for $n \rightarrow+\infty$ and $n^{131 / 416+\varepsilon} \ll h(n)=o(n)$, that is

$$
F(n)=\frac{\sum_{n<k \leqslant n+h(n)} d(k)}{h(n) \log n} \sim 1
$$

for $n \rightarrow+\infty$. To date, this is the best known unconditional result about the above asymptotic formula, as remarked by M. Z. Garaev, F. Luca and W. G. Nowak [5]. We notice that M. Jutila [11] conjectured that

$$
\Delta(x+h)-\Delta(x) \ll \sqrt{h} x^{\varepsilon},
$$

for $x^{\varepsilon} \ll h \ll x^{1 / 2-\varepsilon}$, which is close to being best possible in view of the omega result

$$
\Delta(x+h)-\Delta(x)=\Omega\left(\sqrt{h} \log ^{3 / 2}\left(\frac{\sqrt{x}}{h}\right)\right),
$$

valid for $T \leqslant x \leqslant 2 T, T^{\epsilon} \leqslant h=h(T) \leqslant T^{1 / 2-\epsilon}$, see A. Ivić [10, Corollary 2] and recalling that $f(x)=\Omega(g(x))$ means that $\lim _{x \rightarrow+\infty} f(x) / g(x) \neq 0$. If we assume this strong conjecture one easily obtains that the asymptotic formula (6.4) holds for every $h \gg n^{\varepsilon}$, since for the large values of $h$ the validity of the asymptotic formula is insured by the cited result of M. N. Huxley. Note that the asymptotic formula (6.4) can be proved assuming weaker hypotheses than that of Jutila, see D. Bazzanella [4].

Suppose now that the asymptotic formula (6.4) does not hold with some function $h(x)$ constant on intervals $X \leqslant x<2 X$, where $X$ are of the form $2^{r}$ for $r$ integers, and value $h(x)=X^{\theta}$ for some positive $\theta<131 / 416$. In order to use Theorem 4.14 or Theorem 4.15 we need to verify that $\lim _{n \rightarrow+\infty}[F(n+1)-F(n)]=0$ and that either $1 \in L_{F}$ or (4.7) holds. By reductio ad absurdum, if we assume that $1 \notin L_{F}$ and that (4.7) does not hold, we must have

$$
\liminf _{n \rightarrow+\infty} F(n)>1 \quad \text { or } \quad \limsup _{n \rightarrow+\infty} F(n)<1 .
$$

This implies that

$$
\sum_{n<k \leqslant n+h(n)} d(k)-h(n) \log n+R(n) \gg h(n) \log n
$$

for every function $R(n)=o(h(n) \log n)$. Then we can deduce

$$
\sum_{X \leqslant n \leqslant X+Y}\left|\sum_{n<k \leqslant n+h(n)} d(k)-h(n) \log n+R(n)\right|^{2} \gg Y[h(X)]^{2} \log ^{2} X,
$$


for every function $R(n)=o(h(n) \log n)$ and positive $Y$. Let

$$
\Sigma(n)=\Delta(n+h(n))-\Delta(n)-\left(\sum_{n<k \leqslant n+h(n)} d(k)-h(n) \log n\right)
$$

and observe that definition (6.1) implies

$$
\Sigma(n) \ll h(n)=o(h(n) \log n) .
$$

From the above and (6.7) we have

$$
\sum_{X \leqslant n \leqslant X+Y}\left|\sum_{n<k \leqslant n+h(n)} d(k)-h(n) \log n+\Sigma(n)\right|^{2} \gg Y[h(X)]^{2} \log ^{2} X,
$$

and then

$$
\sum_{X \leqslant n \leqslant X+Y}|\Delta(n+h(n))-\Delta(n)|^{2} \gg Y[h(X)]^{2} \log ^{2} X,
$$

where $h(n)$ is constant in every interval $[X, X+Y] \subseteq[X, 2 X]=\left[2^{r}, 2^{r+1}\right]$. In 1984, M. Jutila [12] proved that

$$
\int_{X}^{X+Y}(\Delta(x+h)-\Delta(x))^{2} \mathrm{~d} x \ll h Y \log ^{3}\left(\frac{\sqrt{X}}{h}\right),
$$

with $X^{\varepsilon} \ll h \leqslant \sqrt{X} / 2$ and $X^{1+\varepsilon} / h \ll Y \leqslant X$, and this yields

$$
\sum_{X \leqslant n \leqslant X+Y}|\Delta(n+h)-\Delta(n)|^{2} \ll h Y X^{\delta}
$$

for arbitrarily small $\delta>0, X^{\varepsilon} \ll h \leqslant \sqrt{X} / 2$ and $X^{1+\varepsilon} / h \ll Y \leqslant X$, since $d(n) \ll n^{\delta}$ for arbitrary $\delta>0$, see T. M. Apostol [1, pag. 296], and then

$$
\Delta(x+h(x))-\Delta(x)=\Delta(n+h(n))-\Delta(n)+O\left(n^{\delta}\right)
$$

for every $x \in[n, n+1]$.

The bound (6.9) is in contradiction with (6.8).

To prove (4.8) we observe that

$$
\begin{aligned}
F(n+1)-F(n)= & \frac{\sum_{n+1<k \leqslant n+1+h(n+1)} d(k)}{h(n+1) \log (n+1)}-\frac{\sum_{n<k \leqslant n+h(n)} d(k)}{h(n) \log n} \\
= & \frac{1}{h(n+1) \log (n+1)} \sum_{n+1<k \leqslant n+1+h(n+1)} d(k) \\
& -\left(1+O\left(\frac{1}{n}\right)\right) \frac{1}{h(n+1) \log (n+1)} \sum_{n<k \leqslant n+h(n)} d(k)
\end{aligned}
$$


and then, using again that $d(n) \ll n^{\delta}$ for arbitrary $\delta>0$, it is simple to verify that (4.8) holds. Now we are able to use either Theorem 4.14 or Theorem 4.15 and this implies that also the asymptotic formula (6.4) does not admit a true exceptional set.

\section{References}

[1] T. M. Apostol, Introduction to analytic number theory, Springer, New York, 1976.

[2] D. Bazzanella and A. Perelli The exceptional set for the number of primes in short intervals, J. Number Theory 80 (2000), 109-124.

[3] D. Bazzanella, Two conditional results about primes in short intervals, Int. J. Number Theory 7 (2011), no. 7, 1753-1759.

[4] D. Bazzanella, On the divisor function in short intervals, Arch. Math. 97 (2011), no. 5, 453-458.

[5] M.Z. Garaev, F. Luca and W.G. Nowak The divisor problem for $d_{4}(n)$ in short intervals, Arch. Math. 86 (2006), 60-66.

[6] D.R. Heath-Brown, The number of primes in a short interval, J. Reine Angew. Math. 389 (1988), 22-63.

[7] M.N. Huxley, On the difference between consecutive primes, Invent. Math. 15 (1972), 164-170.

[8] M.N. Huxley, Exponential sums and lattice points III, Proc. London Math. Soc. 87(3) (2003), 591-609.

[9] A.E. Ingham, On the difference between consecutive primes, Quart. J. of Math. (Oxford) 8 (1937), 255-266.

[10] A. Ivić, On the divisor function and the Riemann zeta-function in short intervals, Ramanujan J. 19 (2009), no. 2, 207-224.

[11] M. Jutila, Riemann's zeta function and the divisor problem, Ark. Mat. 21 (1983), no. 1, 75-96.

[12] M. Jutila, On the divisor problem for short intervals, Ann. Univer. Turkuensis Ser. A.I. 186 (1984), 23-30.

[13] A. S. Kechris, Classical descriptive set theory, Springer, New York, 1995.

[14] H. Ki and T. Linton, Normal numbers and subsets of $\mathbb{N}$ with given densities, Fund. Math. 144 (1994), 163-179.

[15] H. Maier, Primes in short intervals, Michigan Math. J. 32 (1985), 221-225.

[16] A. Selberg, On the normal density of primes in small intervals, and the difference between consecutive primes, Arch. Math. Naturvid. 47 (1943), 87-105.

[17] A. Zaccagnini. Primes in almost all short intervals, Acta Arith. 84 (1998), $225-244$.

Address: Danilo Bazzanella and Riccardo Camerlo: Dipartimento di Scienze Matematiche, Politecnico di Torino, Corso Duca degli Abruzzi 24, 10129 Torino, Italy.

E-mail: danilo.bazzanella@polito.it, camerlo@calvino.polito.it

Received: 8 July 2013; revised: 15 October 2013 